(C) 2020 Copyright held by the owner/author(s). Publication rights licensed to ACM. This is the author's version of the work. It is posted here for your personal use. Not for redistribution. The definitive Version of Record was published in ISS '20 Companion, November 8-1 1, 2020, Virtual Event, Portugal, https://doi.org/10.1 145/3380867.3427410

\title{
Volumetric Reach-through Displays for Direct Manipulation of 3D Content
}

\author{
Iñigo Fermín Ezcurdia
}

\author{
UpnaLab - Public University of Navarre \\ Pamplona, Spain \\ inigofermin.ezcurdia@unavarra.es \\ November 8-11, 2020
}



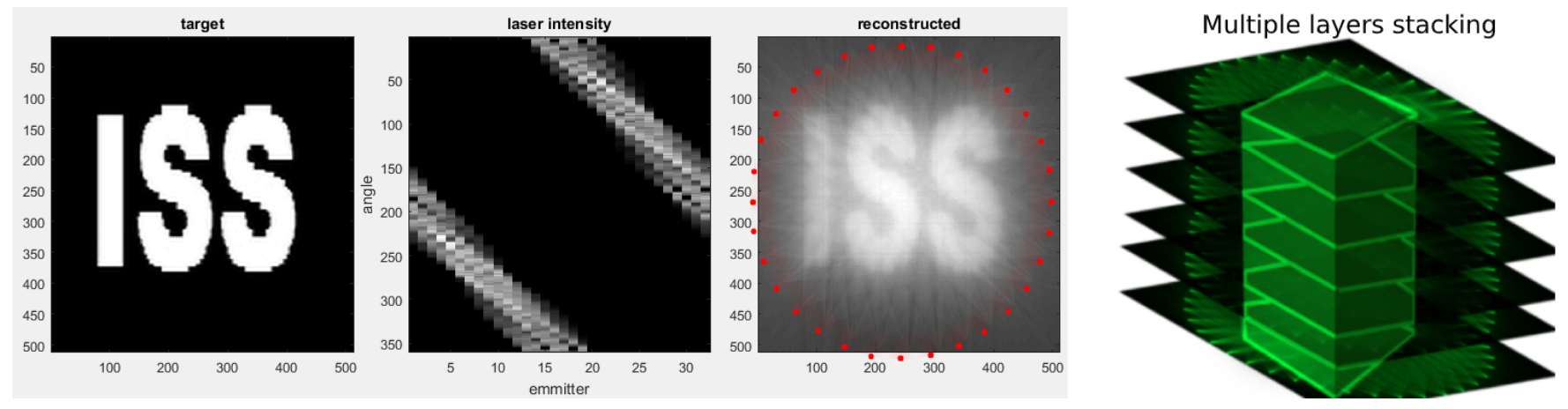

Figure 1: Left) an inverse tomographic display using 32 lasers that scan the display area emitting different intensities for each angle. Right) stacking multiple of these displays to get a reach-through display with multiple depths.

\section{ABSTRACT}

In my PhD, I aim at developing a reach-through volumetric display where points of light are emitted from each $3 \mathrm{~d}$ position of the display volume, and yet it allows people to introduce theirs hands inside to directly interact with the rendered content. Here, I present TomoLit, an inverse tomographic display, where multiple emitters project rays of different intensities for each angle, rendering a target image in mid-air. We have analysed the effect on image quality of the number of emitters, their locations, the angular resolution and the levels of intensities. We have developed a simple emitter and we are in the process of putting together multiple of them. And what I plan to do next, e.g. moving from 2D to 3D and exploring interaction techniques. The feedback obtained in this symposium will clearly dissipate some of of my doubts and guide my research career.

\section{INTRODUCTION}

Science fiction has depicted the so called holographic displays in multiple occasions. These displays are able to render 3D graphics in mid-air . Light is coming from each point of space and these graphics can be observed by multiple people from different points of view, without forcing them to wear any device. There are current realizations of displays that almost achieve this vision, e.g. volumetric displays, light-field displays or levitated tracer particles; to name a few. However, none of them allow people to introduce their hand into the display volume to enable direct and natural interaction with the rendered graphics.

I will explore the novel concept of inverse tomographic displays, getting inspiration from the recent technique of tomographic $3 \mathrm{~d}-$ printing [8] and projection on non-solid diffusers $[13,15,17]$. In regular tomography, multiple sensors can reconstruct the original image from its projections. In our inverse tomographic display, multiple emitters can project from different angles to create a target image on the display area.

\section{RELATED WORK}

A literature review on displays for Anatomical Education [6] exposes the different 3D displays used, their benefits with respect to other alternatives in terms of tools to support learning, spatial perception and user satisfaction. Barry G. Blundell has published various works that review 3D volumetric technologies $[1,2,4]$ as well as evaluating the current state of the art and the needs of future research that this field requires [3]. Given the diversity of options for the creation of volumetric displays and the wide range of possibilities for their characteristics, a division into two categories is proposed [16].

On the one hand, displays that exploit the persistence of human vision to draw different fractions of a 3D object at different steps, so that they are perceived as a single three-dimensional object. To do this, the decomposition of an object into multiple cross sections and its subsequent projection on different surfaces is employed, using LEDs or projection on oscillating or rotating surfaces. We also find solutions that propose the use of mirrors and varifocal lenses projecting at different depths.

On the other hand, we find those displays that create static 3D volumes, without the need for moving parts present in the projected volume itself. In this case we find solutions that propose the use of powerful lasers to generate visible radiation in solids, liquids or gases; exploiting the plasma generation at the focal point [11]. There are special gasses and liquids that only emit light when irradiated by two different wavelengths, this enables to create a 3D image by scanning the two types of light from perpendicular directions [12].

An additionally third category uses acoustically or optically levitated particles fastly move along the path to be rendered [5, 7, 14] or just use them to represent vertices $[9,10]$; however, as soon as an object enters the display volume it disturbs the particle trajectory or collides with it.

The different volumetric displays proposed to date do not allow users to introduce their hands into the display area. 

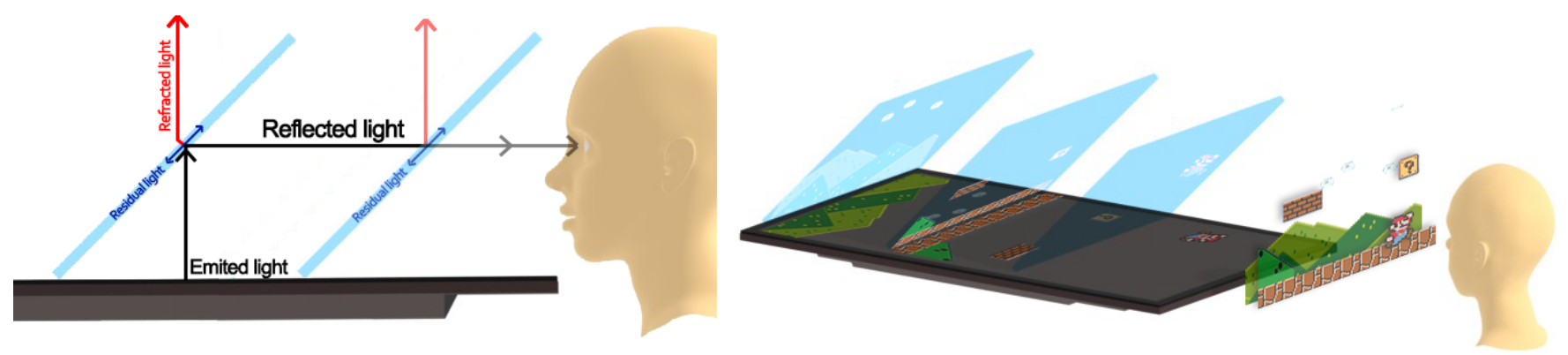

Figure 2: Left) Light reflection, light refraction and residual light on each SliceView's layer. Right) Schematic diagram of a SliceView rendering a layered videogame.

\section{RESEARCH OBJECTIVE}

Volumetric displays render 3D content, without the need of wearing any device such as helmets or glasses. In addition, these displays allow a correct accommodation of the vision focus and the observation by different users from different points of view. However, it is not possible to insert the hand or other object directly into the display area, thus direct interaction is not possible. Direct interaction is a more natural way of interacting with digital content when the interaction space overlaps with the display space. The clearest example is the multi-touch screens of our mobile, where we touch directly with the finger on the buttons or texts with which we want to interact.

The questions that I will try answer in my PhD are:

- What types of volumetric displays exist and what limitations do they have in terms of direct interaction?

- What alternatives can be considered for creating a volumetric display that allows direct interaction?

- What novel possibilities would a volumetric reach-through display enable in terms of interaction?

- How this will change the world?

\section{WHAT HAS BEEN DONE: SLICEVIEW}

SliceView has been my first research project. A first step to acquire basic knowledge about volumetric displays and to improve my research skills. It also has a Do-it-Yourself component that would permit me to present it at maker faires. The optical illusion known as "Pepper's Ghost" consists of placing a plane of a translucent material at a certain angle on a light source, so that it reflects towards the user the projection of an image that is to be superimposed on the reality perceived by the user. SliceView extends this technique to multiple planes, cumulatively giving volume to the initially two-dimensional projection.

A technical evaluation of light reflection, light transmission, and layer thickness was conducted. User studies compared SliceView with a commercial lightfield display in tasks that require observation of information at multiple depths. Furthermore, possible applications were proposed and implemented: A layer-based painting tool, a retro-gaming platform, a text overlaying media player for subtitles, a browser-based 3D scene renderer, and a volumetric data visualizer.

\section{TOMOLIT AND THE CONCEPT OF INVERSE TOMOGRAPHIC DISPLAYS}

We are working on the concept of inverse tomographic displays for rendering in mid-air graphics of one dimensionality higher than its emitting parts. Around the display area or volume, there are emitters that project rays with programmed intensity for each direction, the intersection of the rays produce the graphics in laminar steam or fog.

Currently, we aim to develop a 2D prototype capable of rendering shapes and characters. We have taken inspiration from a Hackio concept [18] which proposes to use lasers and spinning mirrors. We have implemented the concept using low-cost off-the-shelf components. Figure 1.left shows the application of inverse tomography to generate an image with 32 scanning lasers around a circle.

The tomographic technique can be used to find out what intensity each laser has to emit at each angle in order to create the central image (Figure 1.center). Our idea is to make a stack of these displays to be able to create images in different planes (Figure 1.right). The user could put his hand inside these circles to interact directly with the content.

An appropriate scattering medium is required for the correct functioning of this display. We seek for a material that scatters the laser beams with minimum diffusion. We have tested the additive behaviour of the lasers in ultrasonically-generated fog (Figure 3), the addition is not linear but follows the rule brightness = luminosity ${ }^{\frac{1}{3}}$, this will make the images less clear but as it can be seen in the simulations, the rendered images can still be perceived. Solid scattering media such as semi-transparent sponges or fibbers can be used to quickly test results without the cumbersome use of fog although they do not enable reach-through interactions. Laminar flow of steam or dry ice mist would be our preferred scattering medium. 

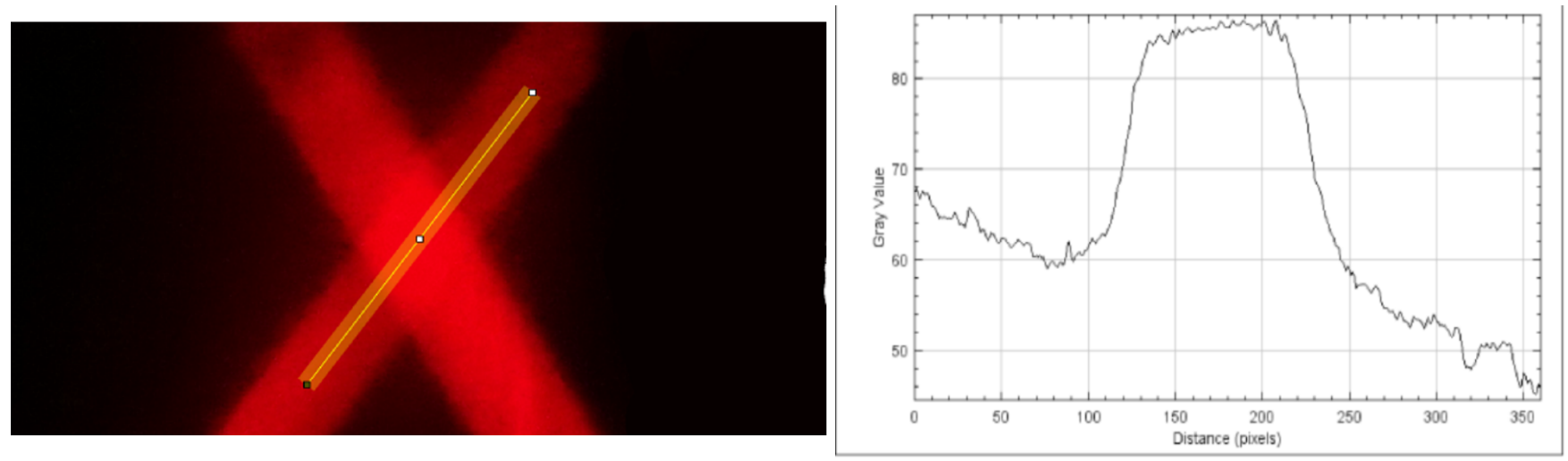

Figure 3: Combined brightness of colliding lasers on mist generated with an ultrasonic nebulizer. The addition is not linear but still presents an accumulative behaviour, which is vital for the working of inverse tomographic displays.

Several simulations have been made to test the optimal number of emitters, intensity levels and angular resolution needed to render recognisable 2D images. Our simulations in Figure 4 suggest that a first prototype using 16 emitters, 16 intensity levels and $1^{\circ}$ of angular resolution offers a good trade-off between image quality and system complexity.

Additional simulations have been made to test the results when the emitters are placed at different locations (Figure 5). The circular arrangement provides the best image quality but the other geometries have interesting features. A geometry open on the top would provide easier access to the display area. The line geometry is the closest to a planar emitter projecting something three-dimensional on top of it. The fact that the semi-circle produces correct images, implies that even when our hand or other objects are inserted in the display area, images will still be rendered around them. This enables multiple opportunities for augmented reality.

Our first emitter prototype (Figure 6) employs a low-intensity laser reflecting on a small rotating mirror. This mirror is glued on a small disc attached to a DC motor. The disc has a small opening that trigger an open optocoupler, this is used to have a reference point of the spinning and disc and to measure the rotational speed. Thereby, the laser intensity can be adjusted for each projection angle and it is stable despite small changes on the motor speed. An Arduino Nano is used as the microcontroller. All the components are contained in a 3D printed structure, This prototype spins 60 times per second, has an angular resolution of $0.35^{\circ}$ and has 60 intensity levels. The ray intensity for each angle is stored in a buffer of 256 positions; using the standard Arduino Serial port, we estimate that we can update the buffer of 32 emitters at 24 frames per second.

\section{FUTURE WORK}

Lightfield displays can be used as 2D emitters, they would surround the display volume to apply the inverse tomographic display principle but this time generating a 3D image with more resolution. Although we expect difficulties due to low-light intensity and the alignment of the lightfield displays. I would also like to explore the direct manipulations that can be done with this technology (Figure 7.left) as well as its augmented reality capabilities to render around the hand and other objects (Figure 7.right).

\section{OPEN QUESTIONS AND ISSUES FOR DISCUSSION}

I would like to discuss different scattering materials to be used for this display. New hardware ideas and improvements would be greatly appreciated. In addition, feedback and suggestions about upgrading from one-layered 2D display to a multiple-layer 3D volumetric display would provide me some guidance for the next steps of this project. Finally, I think the community would suggest interesting and useful applications for this display technology.

\section{ACKNOWLEDGMENTS}

We thank Sonia Elizondo for her simulations and Xabier Galar for the mechanical design of the emitter and the Arduino code; Asier Marzo is supervising the project. This work has been funded by Government of Navarre (FEDER) 0011-1365-2019-000086; and by Jovenes Investigadores UPNA PJUPNA1923.

\section{REFERENCES}

[1] Barry Blundell. 2011. About 3D volumetric displays. Walker \& Wood.

[2] Barry G Blundell. 2010. 3D Displays and Spatial Interaction: Exploring the Science, Art, Evolution and Use of. (2010).

[3] Barry G Blundell. 2017. On the uncertain future of the volumetric 3D display paradigm. 3D Research 8, 2 (2017), 11.

[4] Barry G Blundell and Adam J Schwarz. 2002. The classification of volumetric display systems: characteristics and predictability of the image space. IEEE Transactions on Visualization and Computer Graphics 8, 1 (2002), 66-75. 

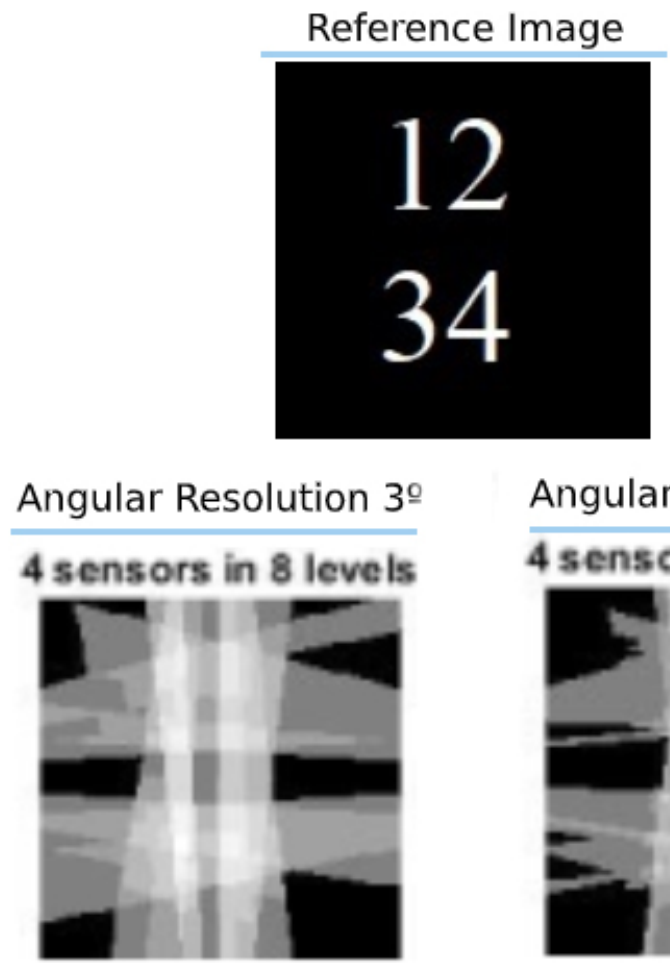

Angular Resolution 10

8 sensors in 16 levels 4 sensors in 8 levels
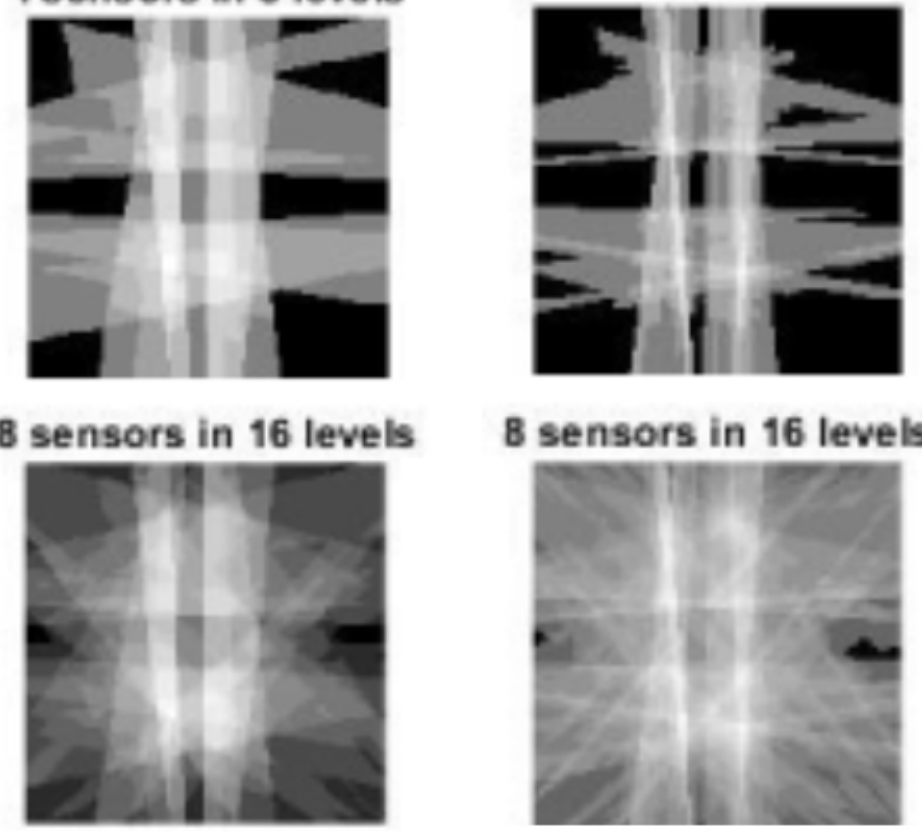

16 sensors in 16 levels
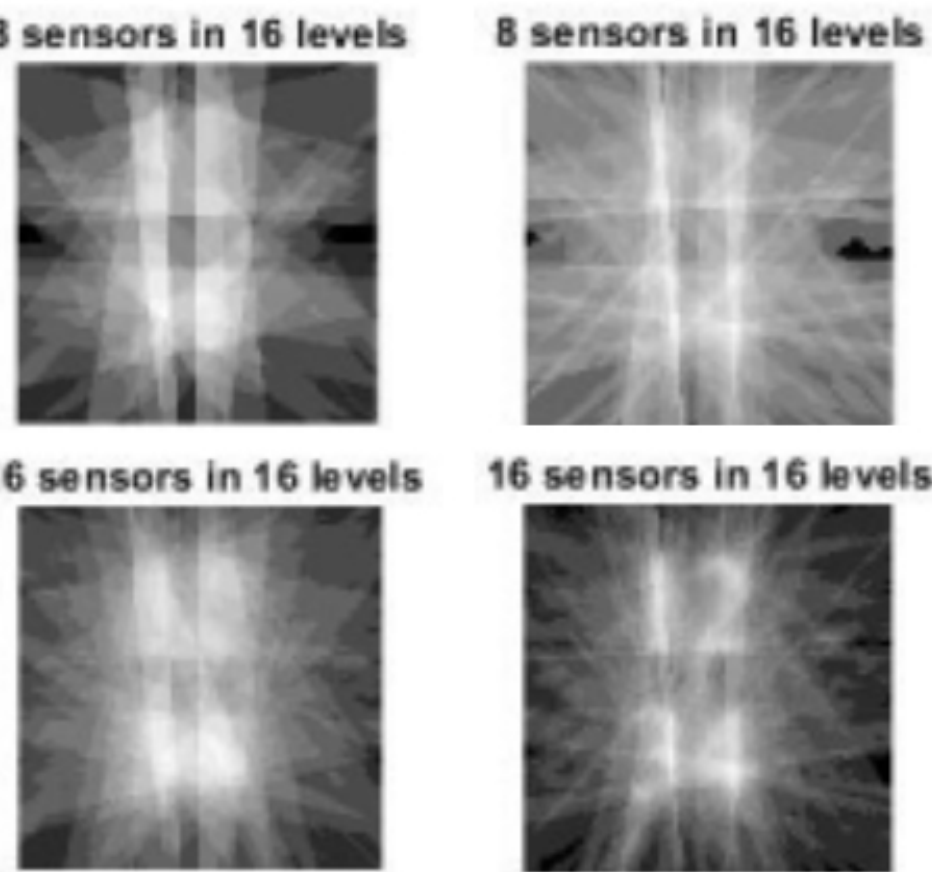

16 sensors in 16 levels

64 sensors in 64 levels
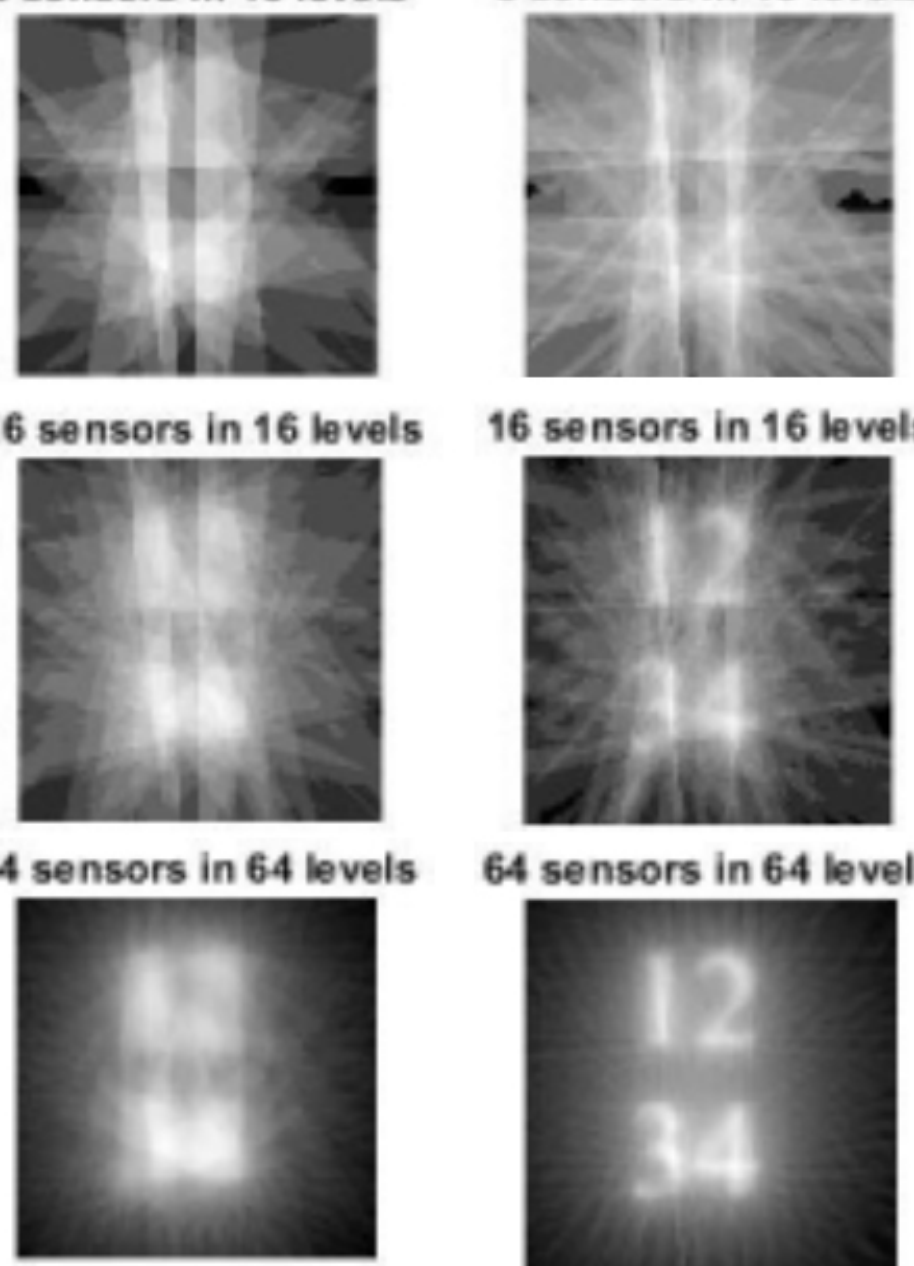

64 sensors in 64 levels

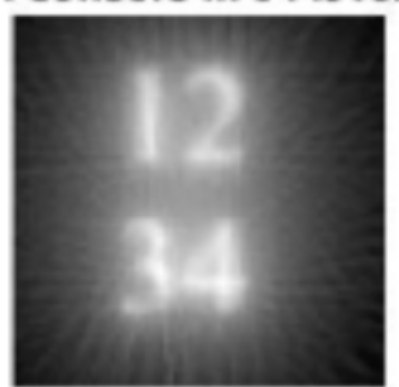

Figure 4: Simulated resulting images for different number of emitters, intensity levels and angular resolution. 

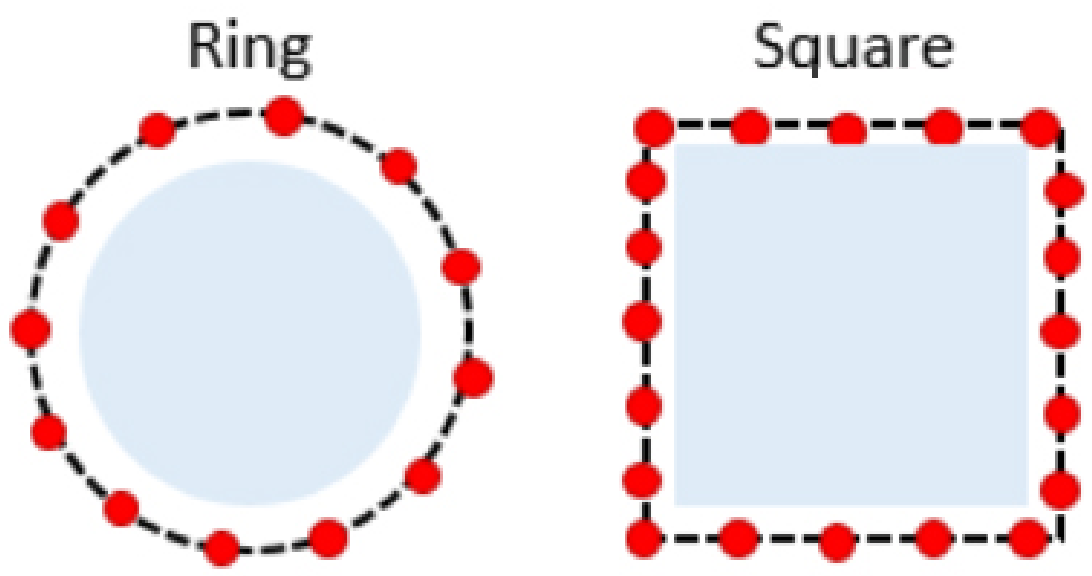

Half-circle
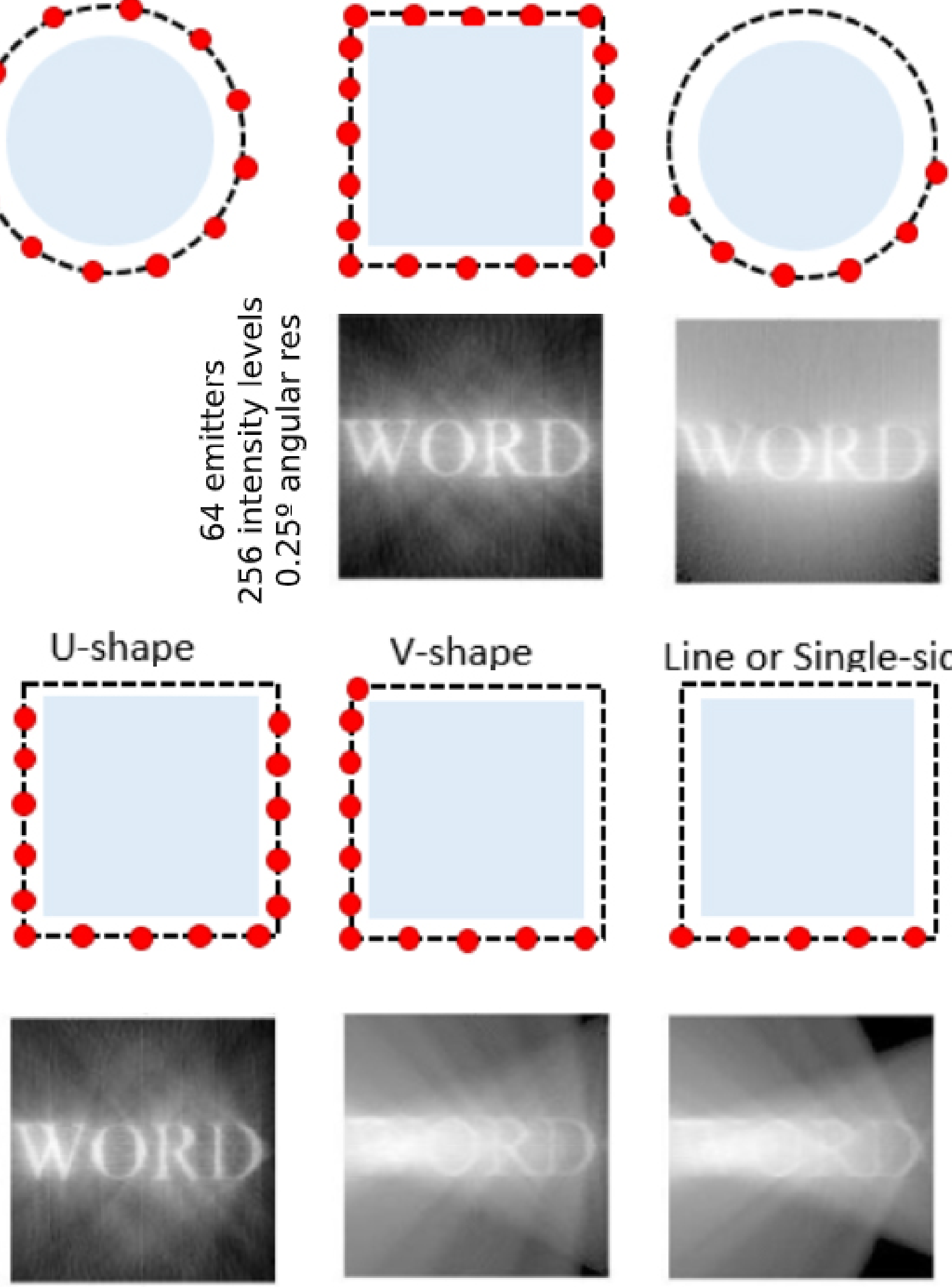

Line or Single-sided
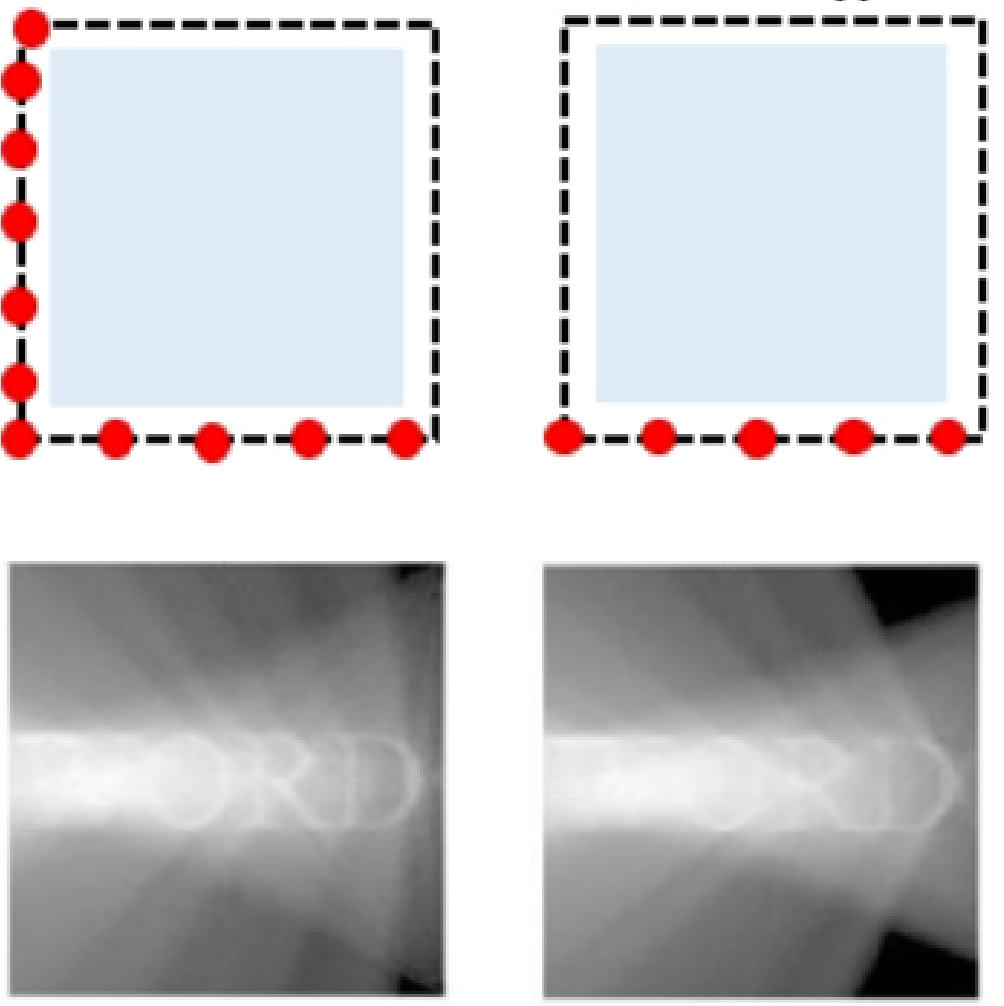

Figure 5: Resulting images for different locations of the emitters. 

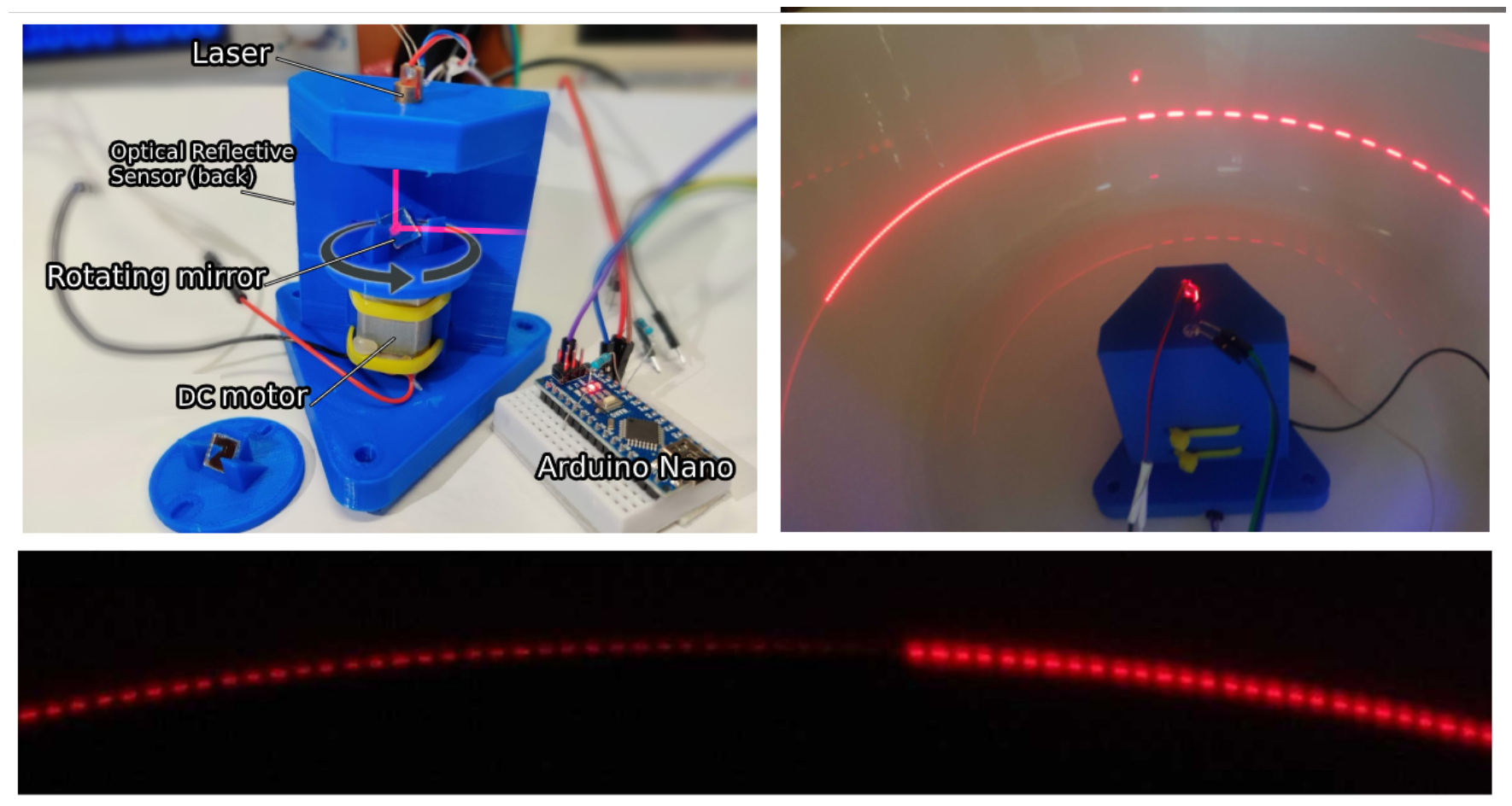

Figure 6: Top-left) Hardware prototype for one emitter. Top-right) Emitter projecting different dashed patterns along 180 $^{\circ}$. Bottom) High-precision projection pattern with increasing and decreasing intensities.
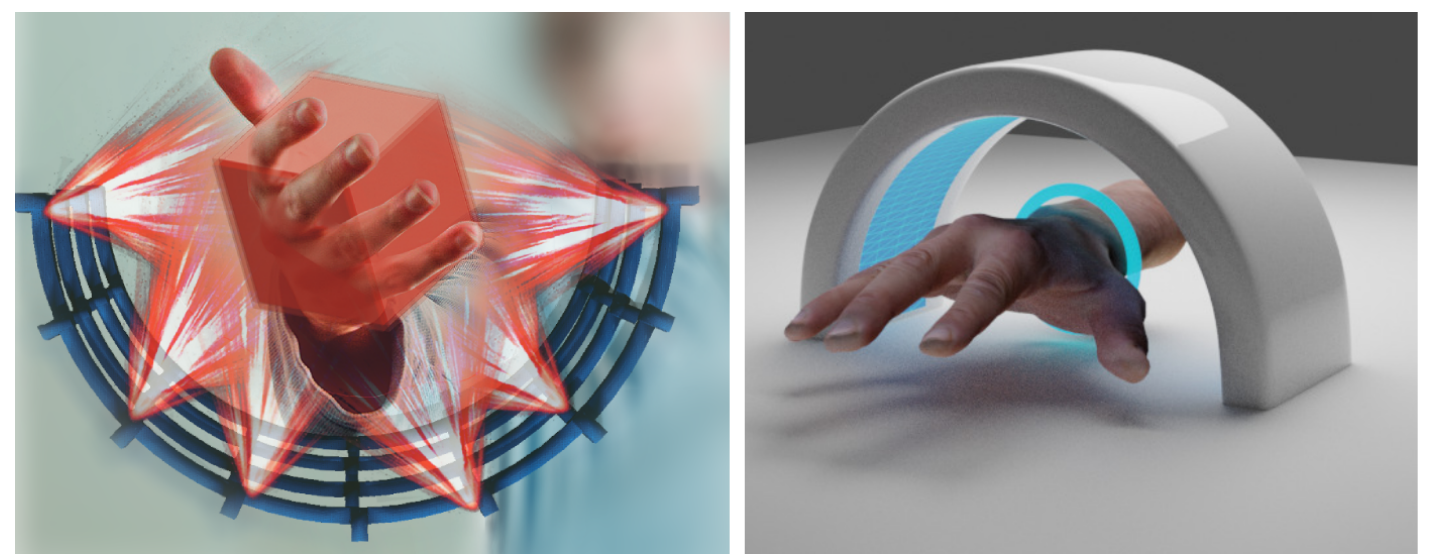

Figure 7: Concept images for a fully developed tomolit displays. Left) Reach-through volumetric half-circle rendering a cube. Right) A bracelet rendered around the user wrist.

[5] Tatsuki Fushimi, Asier Marzo, Bruce W Drinkwater, and Thomas L Hill. 2019. Acoustophoretic volumetric displays using a fast-moving levitated particle. Applied Physics Letters 115, 6 (2019), 064101

[6] Matthew Hackett and Michael Proctor. 2016. Three-dimensional display technologies for anatomical education: a literature Review. Journal of Science Education and Technology 25,4 (2016), 641-654.

[7] Ryuji Hirayama, Diego Martinez Plasencia, Nobuyuki Masuda, and Sriram Subramanian. 2019. A volumetric display for visual, tactile and audio presentation using acoustic trapping. Nature 575, 7782 (2019), 320-323.

[8] Brett E Kelly, Indrasen Bhattacharya, Hossein Heidari, Maxim Shusteff, Christopher M Spadaccini, and Hayden K Taylor. 2019. Volumetric additive manufacturing via tomographic reconstruction. Science 363, 6431 (2019), 1075-1079.

[9] Asier Marzo and Bruce W Drinkwater. 2019. Holographic acoustic tweezers. Proceedings of the National Academy of Sciences 116, 1 (2019), 84-89.

[10] Rafael Morales, Asier Marzo, Sriram Subramanian, and Diego Martínez. 2019. LeviProps: Animating Levitated Optimized Fabric Structures using Holographic Acoustic Tweezers. In Proceedings of the 32nd Annual ACM Symposium on User Interface Software and Technology. 651-661. 
[11] Yoichi Ochiai, Kota Kumagai, Takayuki Hoshi, Jun Rekimoto, Satoshi Hasegawa, and Yoshio Hayasaki. 2016. Fairy lights in femtoseconds: aerial and volumetric graphics rendered by focused femtosecond laser combined with computational holographic fields. ACM Transactions on Graphics (TOG) 35, 2 (2016), 1-14.

[12] Shreya K Patel, Jian Cao, and Alexander R Lippert. 2017. A volumetric three-dimensional digital light photoactivatable dye display. Nature communications 8 (2017), 15239.

[13] Ismo Rakkolainen. 2008. Mid-air displays enabling novel user interfaces. In Proceedings of the 1st ACM international workshop on Semantic ambient media experiences. 25-30.

[14] DE Smalley, E Nygaard, K Squire, J Van Wagoner, J Rasmussen, S Gneiting, K Qaderi, J Goodsell, W Rogers, M Lindsey, et al. 2018. A photophoretic-trap volumetric display. Nature 553, 7689 (2018), 486.

[15] Yutaka Tokuda, Mohd Adili Norasikin, Sriram Subramanian, and Diego Martinez Plasencia. 2017. MistForm: Adaptive shape changing fog screens. In Proceedings of the 2017 CHI Conference on Human Factors in Computing Systems. 4383-4395.

[16] Wikipedia. 2020. Volumetric display - Wikipedia, The Free Encyclopedia. http://en.wikipedia.org/w/index.php?title=Volumetric\%20display\&oldid=964819530. [Online; accessed 01-September-2020].

[17] Asuka Yagi, Masataka Imura, Yoshihiro Kuroda, and Osamu Oshiro. 2011. 360-degree fog projection interactive display. In SIGGRAPH Asia 2011 Emerging Technologies. 1-1.

[18] Ted Yapo. 2016. Mid-air Laser Image Display. https://hackaday.io/project/12889-mid-air-laser-image-display 